\title{
Negative spontaneous emission by a moving two-level atom
}

\author{
Sylvain Lannebère ${ }^{1}$ and Mário G. Silveirinha ${ }^{1,2 *}$ \\ 1 Department of Electrical Engineering, \\ University of Coimbra and Instituto de Telecomunicações, 3030-290 Coimbra, Portugal and \\ ${ }^{2}$ University of Lisbon - Instituto Superior Técnico, \\ Department of Electrical Engineering, 1049-001 Lisboa, Portugal
}

(Dated: October 13, 2018)

\begin{abstract}
In this paper we investigate how the dynamics of a two-level atom is affected by its interaction with the quantized near field of a plasmonic slab in relative motion. We demonstrate that for small separation distances and a relative velocity greater than a certain threshold, this interaction can lead to a population inversion, such that the probability of the excited state exceeds the probability of the ground state, corresponding to a negative spontaneous emission rate. It is shown that the developed theory is intimately related to a classical problem. The problem of quantum friction is analyzed and the differences with respect to the corresponding classical effect are highlighted.
\end{abstract}

\footnotetext{
* To whom correspondence should be addressed: mario.silveirinha@co.it.pt
} 


\section{INTRODUCTION}

Since the pioneering experimental work of Drexhage [1,2], it is well known that an excited atom placed in close vicinity of a planar metallic surface sees its spontaneous emission rate strongly affected by the presence of the surface. This phenomenon plays a role particularly important in modern nano optics and is partly due to the coupling between the atom and the Surface Plasmon Polaritons (SPPs) supported by the metallic surface $[3,4]$. In this paper, we are interested in a related scenario and investigate how the spontaneous emission rate is modified when the metallic surface and the two-level atom are in relative translational motion.

The electromagnetic interactions between polarizable moving matter have been extensively studied in the literature, notably in the context of the dynamical Casimir effect [5-14]. In particular, it was recently proven that two closely separated materials in relative translational motion may start to spontaneously emit light due to the emergence of optical instabilities $[11,13,15]$, an effect related to the Vavilov-Cherenkov radiation but for neutral matter [16]. This phenomenon occurs because of the coupling between the guided modes supported by the moving bodies, and results from the conversion of kinetic energy into electromagnetic energy, originating a quantum friction effect [13, 17-37]. From the quantum mechanical point of view, it can be understood as a consequence of the existence of oscillators associated with negative frequencies (due to the Doppler shift effect) that behave as energy reservoirs, and thereby serve to pump the wave oscillations and generate the unstable behavior [15, 38-41]. Interestingly, similar optical instabilities were predicted to appear for a classical electric dipole moving in the vicinity of a metallic surface supporting surface-plasmon polaritons [38]. The objective of this article is to look at the same problem from the point of view of quantum electrodynamics using the Markov approximation and modeling the point dipole as a two-level atom. It should be mentioned that the friction force and spontaneous emission by a neutral atom interacting with the near-field of a dispersive dielectric in relative motion was studied by different authors (see a review in Refs. [14, 35]). In particular, in Refs. [30, 39] it was shown that a quantum harmonic oscillator initially in its ground state can be excited by the quantized field of a moving dielectric slab, in qualitative

agreement with our general findings. Here, we consider instead that the dipole is a two-level atom and that the slab has a plasmonic response, and connect the negative spontaneous 
emission rate with the unstable response of the corresponding classical problem.

The paper is organized as follows. In Sec. II we describe the geometry of the problem and the adopted formalism. Then, in Sec. III the spontaneous emission rate experienced by the moving atom is determined using the Fermi's golden rule. It is demonstrated that the relative motion may induce non-conservative transitions, which eventually lead to a negative rate of spontaneous emission. In Sec. IV we use classical electrodynamics to find the decay rate of the natural oscillations of a point dipole, and show its relation with the quantum spontaneous emission rate. Then, in section $\mathrm{V}$, the time evolution of the atomic operators expectation is characterized, and it is shown that when the rate of spontaneous emission becomes negative, the system evolves towards an inversion of population. Moreover, it is verified that a quantum friction force emerges in the stationary regime. Finally, in order to illustrate the concepts developed throughout the paper, Sec. VI is devoted to numerical examples in the quasi-static approximation and in particular the conditions to have a negative spontaneous emission are given. The friction force dependence on the atom velocity is also characterized.

\section{THE SYSTEM UNDER-STUDY}

The system studied here is closely related to that considered in Ref. [38] and consists of an electric dipole (modeled as a two-level atom in the quantum case) located at a distance $d$ from a thick metallic surface of infinite extent along the $x$ and $y$ directions, as depicted in Fig. 1.

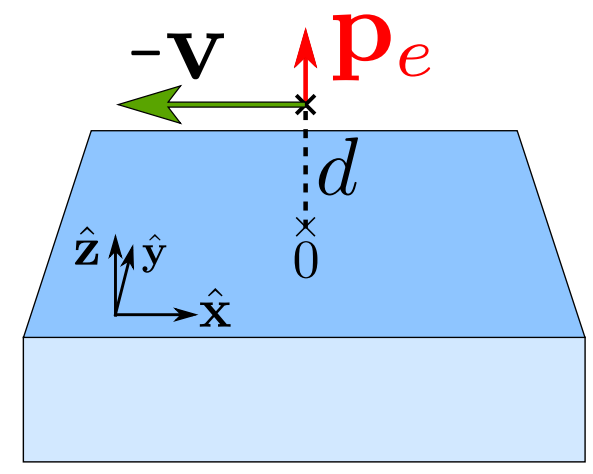

FIG. 1. The system under study: a two-level atom is placed at a distance $d$ from a thick metallic surface. The two-level atom moves with a relative velocity $\mathbf{- v}$ with respect to the metal. 
It is assumed that the permittivity of the metallic slab is described by a lossless Drude model. The relative velocity of the metal slab with respect to the two-level atom is $\mathbf{v}=v \hat{\mathbf{x}}$. Hence, in the frame of the metal slab the two-level atom has velocity $\mathbf{- v}$. It is supposed that the velocity is time-independent, and hence the effect of the optically induced friction-type force - which acts to reduce the relative velocity between the atom and metal - is neglected $[14,35,38]$. It is assumed here and throughout the paper that the unprimed coordinates refer to the reference system co-moving with the slab, and the primed coordinates to the reference system co-moving with the atom. It is also supposed that the relative velocity is significantly smaller than the speed of light in vacuum so that the spacetime coordinates in the two frames can be linked by a Galilean transformation. Moreover, in harmonic regime we adopt the time variation $\mathrm{e}^{-i \omega t}$ for the electromagnetic fields.

\section{A. Quantized electromagnetic field}

As is well known, the quantized electromagnetic field is obtained by associating to each normal mode of the classical problem a quantum harmonic oscillator. For the system of interest, the electromagnetic modes satisfy:

$$
\hat{N} \mathbf{F}=\omega \mathbf{M} \cdot \mathbf{F}
$$

where $\mathbf{F}=\left(\begin{array}{ll}\mathbf{E} & \mathbf{H}\end{array}\right)^{T}$ stands for a six-vector whose components are the electric and the magnetic fields. In the above, $\hat{N}$ is a differential operator

$$
\hat{N}=\left(\begin{array}{cc}
0 & i \nabla \times \mathbf{1}_{3 \times 3} \\
-i \nabla \times \mathbf{1}_{3 \times 3} & 0
\end{array}\right),
$$

and $\mathbf{M}$ is the material matrix

$$
\mathbf{M}(\mathbf{r}, \omega)=\left(\begin{array}{cc}
\varepsilon(\mathbf{r}, \omega) \mathbf{1}_{3 \times 3} & \mathbf{0} \\
\mathbf{0} & \mu_{0} \mathbf{1}_{3 \times 3}
\end{array}\right)
$$

being $\varepsilon$ the space-dependent dispersive permittivity of the system. The relevant materials are assumed non-magnetic. In our problem the system is invariant to translations along the $x$ and $y$ directions and hence the transverse wave vector $\mathbf{k}=\left(k_{x}, k_{y}, 0\right)$ determines two good quantum numbers. Hence, the electromagnetic modes are of the form $\mathbf{F}_{n \mathbf{k}}(\mathbf{r})=\mathbf{f}_{n \mathbf{k}}(z) \mathrm{e}^{i \mathbf{k} \cdot \mathbf{r}}$ 
where $\mathbf{f}_{n \mathbf{k}}$ is the field envelope which depends only on the $z$ coordinate. Then, the quantized fields in the frame co-moving with the plasmonic slab can be written as [42-45]:

$$
\hat{\mathbf{F}}(\mathbf{r}, t)=\sum_{\omega_{n \mathbf{k}}>0} \sqrt{\frac{\hbar \omega_{n \mathbf{k}}}{2}}\left(\hat{a}_{n \mathbf{k}}(t) \mathbf{F}_{n \mathbf{k}}(\mathbf{r})+\hat{a}_{n \mathbf{k}}^{\dagger}(t) \mathbf{F}_{n \mathbf{k}}^{*}(\mathbf{r})\right)
$$

where the sum is restricted to positive oscillation frequencies $\omega_{n \mathbf{k}}$ and $\hat{a}_{n \mathbf{k}}$ and $\hat{a}_{n \mathbf{k}}^{\dagger}$ are the photon annihilation and creation operators for the mode $n \mathbf{k}$, which obey the commutation relation $\left[\hat{a}_{n \mathbf{k}}, \hat{a}_{m \mathbf{q}}^{\dagger}\right]=\delta_{n, m} \delta_{\mathbf{q}, \mathbf{k}}$. In these conditions the field Hamiltonian is

$$
\hat{H}_{\text {field }}=\sum_{\omega_{n \mathbf{k}}>0} \frac{\hbar \omega_{n \mathbf{k}}}{2}\left(\hat{a}_{n \mathbf{k}}^{\dagger} \hat{a}_{n \mathbf{k}}+\hat{a}_{n \mathbf{k}} \hat{a}_{n \mathbf{k}}^{\dagger}\right) .
$$

For dispersive materials the electromagnetic modes must be normalized as:

$$
\left\langle\mathbf{F}_{n \mathbf{k}} \mid \mathbf{F}_{n \mathbf{k}}\right\rangle=\frac{1}{2} \int d^{3} \mathbf{r} \mathbf{F}_{n \mathbf{k}}^{*} \cdot \frac{\partial[\omega \mathbf{M}]}{\partial \omega} \cdot \mathbf{F}_{n \mathbf{k}}=1
$$

This normalization condition is consistent with Ref. [44]. We note in passing that the time-dependent Maxwell equations in dispersive media can always be transformed into a generalized system with no dispersion using the formalism developed in Ref. [46] (see also Appendix B). In this context, the material dispersion is described by additional variables representing the internal degrees of freedom of the material [46-48]. Interestingly, the transformed problem can be readily quantized using standard techniques [45] because the corresponding spectral problem is a standard linear Hermitian eigenvalue problem. In particular, in this framework the normalization condition (6) emerges naturally from Eq. (10) of Ref. $[46]$.

\section{B. Interaction with the two-level atom}

In the quantum description the electric dipole is modeled as a two-level atom whose excited and ground states $|e\rangle$ and $|g\rangle$ are separated by the energy $E_{e}-E_{g}=\hbar \omega_{0}$, where $\omega_{0}$ is the atomic transition frequency. By choosing the energy origin at $\left(E_{e}+E_{g}\right) / 2$, the Hamiltonian of the atom can be written as

$$
\hat{H}_{\mathrm{at}}=\frac{\hbar}{2} \omega_{0} \hat{\sigma}_{z}
$$

with $\hat{\sigma}_{z}=|e\rangle\langle e|-| g\rangle\langle g|$ the atomic inversion operator. 
For low field intensities and a wavelength far greater than the Bohr radius, the Hamiltonian representing the interaction between the two-level atom and the quantized field can be expressed in the dipole approximation as $\hat{H}_{\mathrm{int}}=-\hat{\mathbf{p}}_{e} \cdot \hat{\mathbf{E}}^{\prime}\left(\mathbf{r}_{0}^{\prime}\right)[49]$, where $\hat{\mathbf{E}}^{\prime}\left(\mathbf{r}_{0}^{\prime}\right)$ is the electric field evaluated at the two-level atom position $\left(\mathbf{r}_{0}^{\prime}\right)$ in the atom co-moving frame, and $\hat{\mathbf{p}}_{e}$ is the electric dipole moment operator given by $\hat{\mathbf{p}}_{e}=-e \hat{\mathbf{r}}$. If the states $|e\rangle$ and $|g\rangle$ have opposite parities, then the odd operator $\hat{\mathbf{r}}$ has only non-diagonal components in the $(|e\rangle,|g\rangle)$ basis and the dipole moment is therefore of the form $\hat{\mathbf{p}}_{e}=\boldsymbol{\gamma}_{e g}^{*} \hat{\sigma}_{+}+\boldsymbol{\gamma}_{e g} \hat{\sigma}_{-}$. Here, $\hat{\sigma}_{+}=|e\rangle\langle g|$ describes a transition to the excited state, $\hat{\sigma}_{-}=|g\rangle\langle e|$ describes a transition to the ground state, and the vector $\gamma_{e g}$ represents the transition electric dipole moment.

Throughout this paper the non-relativistic regime is assumed so that the electromagnetic fields in the two reference frames are simply related by $\hat{\mathbf{F}}^{\prime}\left(\mathbf{r}_{0}^{\prime}, t^{\prime}\right) \approx \hat{\mathbf{F}}\left(\mathbf{r}_{0}, t\right)$ with $\mathbf{r}_{0}=\mathbf{r}_{0}^{\prime}-\mathbf{v} t$ [50]. Then, with these approximations and using the six-vector formalism the interaction Hamiltonian can be written as

$$
\hat{H}_{\mathrm{int}} \approx-\left(\tilde{\gamma}_{e g}^{*} \hat{\sigma}_{+}+\tilde{\gamma}_{e g} \hat{\sigma}_{-}\right) \cdot \hat{\mathbf{F}}\left(\mathbf{r}_{0}^{\prime}-\mathbf{v} t\right),
$$

where $\tilde{\gamma}_{e g}=\left(\begin{array}{ll}\gamma_{e g} & 0\end{array}\right)^{T}$ is a six-vector. Using the expression of the quantized field (4) and taking into account the dependence of classical fields in the transverse $(x$ and $y)$ coordinates it follows that:

$$
\hat{H}_{\mathrm{int}} \approx-\left(\tilde{\gamma}_{e g}^{*} \hat{\sigma}_{+}+\tilde{\gamma}_{e g} \hat{\sigma}_{-}\right) \cdot \sum_{\omega_{n \mathbf{k}}>0} \sqrt{\frac{\hbar \omega_{n \mathbf{k}}}{2}}\left(\hat{a}_{n \mathbf{k}} \mathbf{F}_{n \mathbf{k}}\left(\mathbf{r}_{0}^{\prime}\right) \mathrm{e}^{-i \mathbf{k} \cdot \mathbf{v} t}+\hat{a}_{n \mathbf{k}}^{\dagger} \mathbf{F}_{n \mathbf{k}}^{*}\left(\mathbf{r}_{0}^{\prime}\right) \mathrm{e}^{i \mathbf{k} \cdot \mathbf{v} t}\right)
$$

It is important to note that this Hamiltonian induces four types of transitions. Two transitions are associated with the so-called energy conserving terms (proportional to $\hat{\sigma}_{+} \hat{a}_{n \mathbf{k}}$ or $\left.\hat{\sigma}_{-} \hat{a}_{n \mathbf{k}}^{\dagger}\right)$, and the other two transitions describe non-conservative processes that increase $\left(\hat{\sigma}_{+} \hat{a}_{n \mathbf{k}}^{\dagger}\right)$ or decrease $\left(\hat{\sigma}_{-} \hat{a}_{n \mathbf{k}}\right)$ both quantum numbers. As shown in the following sections, the non-conservative processes - which play no role when the relative velocity vanishes can become dominant for large velocities and small separation distances.

\section{QUANTUM SPONTANEOUS EMISSION RATE}

Next, the Fermi's golden rule is used to obtain the spontaneous emission rate experienced by the two-level atom when moving in the vicinity of the plasmonic slab. According to the Fermi's golden rule [51], the number of transitions per unit time $R_{|i\rangle \rightarrow|f\rangle}$ from an initial state 
$|i\rangle$ with energy $E_{i}=\hbar \omega_{i}$ to a final state $|f\rangle$ with energy $E_{f}=\hbar \omega_{f}$ can be expressed as

$$
R_{|i\rangle \rightarrow|f\rangle}=\lim _{t \rightarrow \infty} \frac{\left|\int_{0}^{t}\left\langle f\left|\hat{H}_{\mathrm{int}}\right| i\right\rangle \mathrm{e}^{i\left(\omega_{f}-\omega_{i}\right) t^{\prime}} d t^{\prime}\right|^{2}}{\hbar^{2} t},
$$

where the states are assumed to belong to a continuum. We are interested in two competing processes. The first process is the usual spontaneous emission transition between the excited state of the atom to an excited state of the field with a single photon: $|e, 0\rangle \rightarrow\left|g, 1_{n \mathbf{k}}\right\rangle$ (here the second index refers to the field state). Using the interaction Hamiltonian (9) in Eq. (10) and summing over all possible radiation channels it is found that the transition rate for this process is

$$
\Gamma^{+}=\sum_{\omega_{n \mathbf{k}}>0} \frac{\pi}{\hbar} \omega_{n \mathbf{k}}\left|\tilde{\gamma}_{e g} \cdot \mathbf{F}_{n \mathbf{k}}^{*}\left(\mathbf{r}_{0}^{\prime}\right)\right|^{2} \delta\left(\omega_{0}-\omega_{n \mathbf{k}}^{\prime}\right),
$$

where $\omega_{n \mathbf{k}}^{\prime}=\omega_{n \mathbf{k}}+\mathbf{k} \cdot \mathbf{v}$ is the Doppler shifted frequency in the reference frame of the two-level atom. Evidently, the described mechanism drives the atom to its ground state.

The second process of relevance corresponds to a transition between the ground state of the atom to an excited state of the field: $|g, 0\rangle \rightarrow\left|e, 1_{n \mathbf{k}}\right\rangle$. A priori this process may seem impossible because $|g, 0\rangle$ is the ground state of the non-interacting atom and field, but a simple calculation shows that its time rate is determined by:

$$
\Gamma^{-}=\sum_{\omega_{n \mathbf{k}}>0} \frac{\pi}{\hbar} \omega_{n \mathbf{k}}\left|\tilde{\gamma}_{e g}^{*} \cdot \mathbf{F}_{n \mathbf{k}}^{*}\left(\mathbf{r}_{0}^{\prime}\right)\right|^{2} \delta\left(\omega_{0}+\omega_{n \mathbf{k}}^{\prime}\right) .
$$

Evidently, this process drives the atom (and the field) to an excited state, even though there is no photon absorption. When the relative velocity between the atom and the metal vanishes, $\Gamma^{-}=0$, as expected. However, when there is relative motion the Doppler shifted frequencies $\omega_{n \mathbf{k}}^{\prime}$ may be negative, and hence the relative motion may induce transitions to the excited state.

In general, transitions between $|e, 0\rangle \rightarrow\left|g, 1_{\mathbf{k}}\right\rangle$ involve only positive Doppler shifted frequencies such that $\omega_{n \mathbf{k}}^{\prime}=\omega_{0}$. These transitions are generated by the energy conserving terms in Eq. (9). On the other hand, transitions between $|g, 0\rangle \rightarrow\left|e, 1_{\mathbf{k}}\right\rangle$ involve only negative Doppler shifted frequencies such that $\omega_{n \mathbf{k}}^{\prime}=-\omega_{0}$, and thus it follows that they can only occur when the relative velocity between the slab and the atom is large enough to provide $\omega_{n \mathbf{k}}^{\prime}<0$. Such transitions are induced by the non-conservative terms in the interaction Hamiltonian (9), and the conservation of the total energy in the system implies that they should be associated with a reduction of the kinetic energy [13, 38, 52, 53]. 
We define the total spontaneous emission rate $\Gamma_{\text {gr }}$ from the excited state to the ground, $|e\rangle \rightarrow|g\rangle$, as the difference between the transition rates of the two competing processes:

$$
\begin{aligned}
\Gamma_{\mathrm{gr}} & =\Gamma^{+}-\Gamma^{-} \\
& =\frac{\pi}{\hbar} \sum_{\omega_{n \mathbf{k}}>0} \omega_{n \mathbf{k}}\left[\left|\tilde{\gamma}_{e g} \cdot \mathbf{F}_{n \mathbf{k}}^{*}\left(\mathbf{r}_{0}^{\prime}\right)\right|^{2} \delta\left(\omega_{n \mathbf{k}}^{\prime}-\omega_{0}\right)-\left|\tilde{\gamma}_{e g}^{*} \cdot \mathbf{F}_{n \mathbf{k}}^{*}\left(\mathbf{r}_{0}^{\prime}\right)\right|^{2} \delta\left(\omega_{n \mathbf{k}}^{\prime}+\omega_{0}\right)\right] .
\end{aligned}
$$

The physical meaning of $\Gamma_{\text {gr }}$ will be further elaborated later. Of course, in a situation with no relative motion the contribution of $\Gamma^{-}$vanishes and the spontaneous emission rate $\Gamma_{\text {gr }}$ reduces to the usual result for a dipole located near a stationary metallic surface [4]. Notably, it will be shown ahead that it is possible to find a regime in which $\Gamma^{-}>\Gamma^{+}$i.e. a situation where the interaction with the negative Doppler shifted frequencies is dominant, such that the total spontaneous emission rate becomes negative, implying that the state $|g, 0\rangle$ becomes less likely of being occupied than the state $|e, 0\rangle$.

\section{THE DECAY RATE FOR THE CLASSICAL PROBLEM}

In order to link the spontaneous emission rate $\Gamma_{\text {gr }}$ with a classical result, we note that a neutral two-level atom can be modeled classically as an electric dipole (with dipole moment $\mathbf{p}_{e}$ ) with a polarizability (along the relevant direction of space) given by the semi-classical formula $[38,54]$

$$
\alpha_{e}^{-1}(\omega)=\frac{\hbar \omega_{0} \varepsilon_{0}}{\left|\gamma_{e g}\right|^{2}}\left(\frac{1}{2 \omega_{0}^{2}}\left(\Gamma_{0}^{2}+\omega_{0}^{2}-\omega^{2}\right)-i \Gamma_{0} \frac{\omega}{\omega_{0}^{2}}\right)
$$

where $\omega_{0}$ is the transition frequency of the two-level atom, $\gamma_{e g}$ is the transition dipole moment

and $\Gamma_{0}=\frac{\left|\gamma_{e g}\right|^{2}}{6 \pi \varepsilon_{0} \hbar}\left(\frac{\omega_{0}}{c}\right)^{3}$. When the electric dipole is in the vicinity of the plasmonic slab, the natural frequencies of oscillation, $\omega=\omega^{\prime}+i \omega^{\prime \prime}$, are determined by the solutions of

$$
\gamma_{e g}^{*} \cdot\left[\alpha_{e}^{-1}(\omega)-\underline{C}_{\text {int }}(\omega)\right] \cdot \gamma_{e g}=0,
$$

where $\underline{C}_{\text {int }}$ is an interaction dyadic that relates the local field acting on the dipole with the electric dipole moment: $\mathbf{E}_{\mathrm{loc}}^{\prime}=\underline{C}_{\mathrm{int}} \cdot \mathbf{p}_{e} / \varepsilon_{0}$ [38]. The local field is the total field excluding the self-field radiated by the dipole, and depends on the metal slab and on the relative velocity. The resonances of atomic systems are characterized by large quality factors, and hence the solution of (15) can be obtained with perturbation theory. The solution is of the form $\omega \approx \omega_{0}+i \omega^{\prime \prime}$ with the imaginary part given by [38]:

$$
\omega^{\prime \prime} \approx-\frac{1}{\hbar \varepsilon_{0}} \operatorname{Im}\left\{\gamma_{e g}^{*} \cdot\left[i \frac{1}{6 \pi}\left(\frac{\omega_{0}}{c}\right)^{3}+\underline{C}_{\mathrm{int}}\left(\omega_{0}\right)\right] \cdot \gamma_{e g}\right\} \text {. }
$$


It is convenient to rewrite this result as:

$$
\omega^{\prime \prime} \approx-\frac{1}{\hbar \varepsilon_{0}} \operatorname{Im}\left\{\gamma_{e g}^{*} \cdot \underline{C}_{\mathrm{tot}}\left(\omega_{0}\right) \cdot \gamma_{e g}\right\}
$$

where $\underline{C}_{\text {tot }}$ is the interaction dyadic that relates the total electric field (including the selffield of the dipole) with the electric dipole moment: $\mathbf{E}^{\prime}=\underline{C}_{\text {tot }} \cdot \mathbf{p}_{e} / \varepsilon_{0}$. Noting that the electromagnetic energy is proportional to $\mathrm{e}^{2 \omega^{\prime \prime} t}$, it follows that the classical decay rate of the dipole is $\Gamma_{\mathrm{cl}}=-2 \omega^{\prime \prime}$, or more explicitly

$$
\Gamma_{\mathrm{cl}} \approx \frac{2}{\hbar \varepsilon_{0}} \operatorname{Im}\left\{\gamma_{e g}^{*} \cdot \underline{C}_{\mathrm{tot}}\left(\omega_{0}\right) \cdot \gamma_{e g}\right\}
$$

As expected, in the absence of interactions with the metallic slab $\left(\underline{C}_{\text {int }}=0\right)$, the classical decay rate reduces to the free space spontaneous emission's rate: $\Gamma_{\mathrm{sp}}=\frac{\left|\gamma_{e g}\right|^{2}}{3 \pi \hbar \varepsilon_{0}}\left(\frac{\omega_{0}}{c}\right)^{3}$. Next, we demonstrate that this result is generally valid and that $\Gamma_{\mathrm{cl}}$ is coincident with the quantum spontaneous emission rate $\Gamma_{\text {gr }}$ determined in the previous section [Eq. (13)].

In Appendix A, the field radiated by the moving classical dipole is explicitly calculated based on an eigenmode expansion. Using a non-relativistic approximation, it is shown that in the reference frame co-moving with the dipole the field is determined by Eq. (A11). Let us introduce an $6 \times 6$ interaction dyadic $\underline{C}_{\text {tot }}^{\mathrm{g}}$ defined in such a manner that:

$$
\mathbf{F}^{\prime}\left(\mathbf{r}_{0}^{\prime}\right)=\underline{C_{\mathrm{tot}}^{\mathrm{g}}(\omega) \cdot \mathbf{p}}
$$

where $\mathbf{p}=\left(\begin{array}{ll}\mathbf{p}_{e} & 0\end{array}\right)^{T}$ is the generalized dipole moment (a six-vector). From Eq. (A11), it is clear that:

$\underline{C}_{\text {tot }}^{\mathrm{g}}(\omega)=\sum_{\omega_{n \mathbf{k}}>0} \frac{\omega_{n \mathbf{k}}}{2}\left(\frac{1}{\omega_{n \mathbf{k}}^{\prime}-\omega} \mathbf{F}_{n \mathbf{k}}\left(\mathbf{r}_{0}^{\prime}\right) \otimes \mathbf{F}_{n \mathbf{k}}^{*}\left(\mathbf{r}_{0}^{\prime}\right)+\frac{1}{\omega_{n \mathbf{k}}^{\prime}+\omega} \mathbf{F}_{n \mathbf{k}}^{*}\left(\mathbf{r}_{0}^{\prime}\right) \otimes \mathbf{F}_{n \mathbf{k}}\left(\mathbf{r}_{0}^{\prime}\right)\right)-\mathbf{M}_{0}^{-1} \delta(0)$.

where $\mathbf{M}_{0}$ is the material matrix in the free-space region and the positive frequency eigenmodes are normalized as in Eq.(6). Note that $\underline{C}_{\text {tot }} / \varepsilon_{0}$ is the electric sub-component of $\underline{C}_{\text {tot }}^{\mathrm{g}}$ (the $3 \times 3$ sub-block matrix in the upper-left corner). Using $\frac{1}{\omega_{n} \pm \omega}=\mathcal{P} \frac{1}{\omega_{n} \pm \omega} \mp i \pi \delta\left(\omega_{n} \pm \omega\right)$ (where $\mathcal{P}$ stands for the principal value operator), it follows that the anti-Hermitian part of the interaction dyadic is

$$
\begin{aligned}
& \frac{1}{2 i}\left[\underline{C}_{\mathrm{tot}}^{\mathrm{g}}(\omega)-\underline{C}_{\mathrm{tot}}^{\mathrm{g}}(\omega)^{\dagger}\right]= \\
& \quad \sum_{\omega_{n \mathbf{k}}>0} \frac{\pi \omega_{n \mathbf{k}}}{2}\left[\delta\left(\omega_{n \mathbf{k}}^{\prime}-\omega\right) \mathbf{F}_{n \mathbf{k}}\left(\mathbf{r}_{0}^{\prime}\right) \otimes \mathbf{F}_{n \mathbf{k}}^{*}\left(\mathbf{r}_{0}^{\prime}\right)-\delta\left(\omega_{n \mathbf{k}}^{\prime}+\omega\right) \mathbf{F}_{n \mathbf{k}}^{*}\left(\mathbf{r}_{0}^{\prime}\right) \otimes \mathbf{F}_{n \mathbf{k}}\left(\mathbf{r}_{0}^{\prime}\right)\right]
\end{aligned}
$$


Hence, by direct comparison with the quantum emission rate obtained with the Fermi's golden rule (13) and recalling that $\tilde{\gamma}_{e g}=\left(\begin{array}{ll}\gamma_{e g} & 0\end{array}\right)^{T}$, it is seen that:

$$
\Gamma_{\mathrm{gr}}=\frac{2}{\hbar} \operatorname{Im}\left\{\tilde{\gamma}_{e g}^{*} \cdot \underline{C}_{\mathrm{tot}}^{\mathrm{g}}\left(\omega_{0}\right) \cdot \tilde{\gamma}_{e g}\right\} .
$$

We used the property $\operatorname{Im}\left\{\mathbf{w}^{*} \cdot \mathbf{A} \cdot \mathbf{w}\right\}=\mathbf{w}^{*} \cdot \frac{\mathbf{A}-\mathbf{A}^{\dagger}}{2 i} \cdot \mathbf{w}$. It is evident that the right-hand side of Eqs. (18) and (22) is the same, and hence the desired result $\Gamma_{\mathrm{cl}}=\Gamma_{\mathrm{gr}}$ is demonstrated: the quantum spontaneous emission rate is exactly coincident with the decay rate of the corresponding classical problem.

\section{TIME EVOLUTION OF THE ATOMIC OPERATORS}

To further characterize the dynamics of the two-level atom, in this section we study the time evolution of the atomic operators and find their vacuum expectation values using the Markov approximation. In the Heisenberg picture, the time evolution of an operator $\hat{A}$ that does not depend explicitly on time is

$$
\frac{\mathrm{d} \hat{A}}{\mathrm{~d} t}=\frac{i}{\hbar}[\hat{H}, \hat{A}] .
$$

It is important to note here that even though the interaction Hamiltonian depends explicitly on time the relevant commutation relations are preserved for all $t$.

Using $\hat{H}=\hat{H}_{\text {field }}+\hat{H}_{\text {at }}+\hat{H}_{\text {int }}$ in Eq. (23) it is easily found that the time evolution of the atomic inversion operator is determined by:

$$
\frac{\mathrm{d} \hat{\sigma}_{z}}{\mathrm{~d} t}=\frac{2 i}{\hbar} \hat{\mathbf{F}}\left(\mathbf{r}_{0}^{\prime}-\mathbf{v} t\right) \cdot\left(\tilde{\gamma}_{e g}^{*} \hat{\sigma}_{+}-\tilde{\boldsymbol{\gamma}}_{e g} \hat{\sigma}_{-}\right)
$$

To make additional progress, in the next sub-section we characterize the field operators.

\section{A. Evolution of the field operators}

Using again Eq. (23) and the standard commutation relations, one finds that the annihilation operators satisfy:

$$
\frac{\mathrm{d} \hat{a}_{n \mathbf{k}}}{\mathrm{d} t}=-i \omega_{n \mathbf{k}} \hat{a}_{n \mathbf{k}}+i \sqrt{\frac{\omega_{n \mathbf{k}}}{2 \hbar}} \hat{\mathbf{p}} \cdot \mathbf{F}_{n \mathbf{k}}^{*}\left(\mathbf{r}_{0}^{\prime}-\mathbf{v} t\right),
$$


where we introduced the operator $\hat{\mathbf{p}}=\left(\tilde{\boldsymbol{\gamma}}_{e g}^{*} \hat{\sigma}_{+}+\tilde{\boldsymbol{\gamma}}_{e g} \hat{\sigma}_{-}\right)$. This differential equation can be written in an integral form as:

$$
\hat{a}_{n \mathbf{k}}(t)=\hat{a}_{n \mathbf{k}} e^{-i \omega_{n \mathbf{k}} t}+i \sqrt{\frac{\omega_{n \mathbf{k}}}{2 \hbar}} \mathbf{F}_{n \mathbf{k}}^{*}\left(\mathbf{r}_{0}^{\prime}-\mathbf{v} t\right) \cdot \int_{0}^{t} \hat{\mathbf{p}}\left(t_{1}\right) u\left(t-t_{1}\right) e^{-i \omega_{n \mathbf{k}}^{\prime}\left(t-t_{1}\right)} d t_{1}
$$

where $u$ denotes the Heaviside's step function. To make further progress and obtain a closed form solution we use the Markov's approximation [55]:

$$
\begin{aligned}
\int_{0}^{t} \hat{\mathbf{p}}\left(t_{1}\right) u\left(t-t_{1}\right) e^{-i \omega_{n \mathbf{k}}^{\prime}\left(t-t_{1}\right)} d t_{1} & \approx \tilde{\gamma}_{e g} \hat{\sigma}_{-}(t) \int_{0}^{t} u\left(t-t_{1}\right) e^{-i\left(\omega_{n \mathbf{k}}^{\prime}-\omega_{0}\right)\left(t-t_{1}\right)} d t_{1} \\
& +\tilde{\gamma}_{e g}^{*} \hat{\sigma}_{+}(t) \int_{0}^{t} u\left(t-t_{1}\right) e^{-i\left(\omega_{n \mathbf{k}}^{\prime}+\omega_{0}\right)\left(t-t_{1}\right)} d t_{1} .
\end{aligned}
$$

Moreover, for large $t$ one has the approximate identities

$$
\int_{0}^{t} u\left(t-t_{1}\right) e^{-i \omega\left(t-t_{1}\right)} d t_{1} \approx \int_{-\infty}^{+\infty} u\left(t_{1}\right) e^{-i \omega t_{1}} d t_{1} \approx \pi \delta(\omega)
$$

where the imaginary part of the last integral is dropped. Hence, from here we finally conclude that:

$\hat{a}_{n \mathbf{k}}(t) \approx \hat{a}_{n \mathbf{k}} \mathrm{e}^{-i \omega_{n \mathbf{k}} t}+i \pi \sqrt{\frac{\omega_{n \mathbf{k}}}{2 \hbar}} \mathbf{F}_{n \mathbf{k}}^{*}\left(\mathbf{r}_{0}^{\prime}\right) \mathrm{e}^{i \mathbf{k} \cdot \mathbf{v} t} \cdot\left[\tilde{\gamma}_{e g}^{*} \hat{\sigma}_{+}(t) \delta\left(\omega_{0}+\omega_{n \mathbf{k}}^{\prime}\right)+\tilde{\gamma}_{e g} \hat{\sigma}_{-}(t) \delta\left(\omega_{0}-\omega_{n \mathbf{k}}^{\prime}\right)\right]$.

Here it is worth pointing out that in Ref. [56] it was argued that the Markov approximation might be inadequate to characterize the stationary state regime $(t \rightarrow \infty)$ in the presence of material dissipation. In principle, our theory is not affected by such a result (at least in the quasi-static limit considered later) because we deal with an ideal lossless system.

\section{B. The inversion operator expectation}

To determine the time evolution of the expectation of the inversion operator, it is con-

venient to decompose the electromagnetic field operator as $\hat{\mathbf{F}}=\hat{\mathbf{F}}^{-}+\hat{\mathbf{F}}^{+}$such that the annihilation part is

$$
\hat{\mathbf{F}}^{-}(\mathbf{r}, t)=\sum_{\omega_{n \mathbf{k}}>0} \sqrt{\frac{\hbar \omega_{n \mathbf{k}}}{2}} \hat{a}_{n \mathbf{k}}(t) \mathbf{F}_{n \mathbf{k}}(\mathbf{r})
$$


with $\hat{a}_{n \mathbf{k}}(t)$ given by the approximate expression $(29)$ and $\hat{\mathbf{F}}^{+}=\left(\hat{\mathbf{F}}^{-}\right)^{\dagger}$. Calculating now the expectation of both sides of Eq. (24) and using normal ordering

$$
\hat{\mathbf{F}} \cdot\left(\tilde{\boldsymbol{\gamma}}_{e g}^{*} \hat{\sigma}_{+}-\tilde{\gamma}_{e g} \hat{\sigma}_{-}\right)=\hat{\mathbf{F}}^{+} \cdot\left(\tilde{\boldsymbol{\gamma}}_{e g}^{*} \hat{\sigma}_{+}-\tilde{\gamma}_{e g} \hat{\sigma}_{-}\right)+\left(\tilde{\boldsymbol{\gamma}}_{e g}^{*} \hat{\sigma}_{+}-\tilde{\boldsymbol{\gamma}}_{e g} \hat{\sigma}_{-}\right) \cdot \hat{\mathbf{F}}^{-}
$$

to eliminate the contribution of the free field part of the electromagnetic field (first term in the right-hand side of Eq. (29)) [55], it is found after some simplifications that:

$$
\frac{\mathrm{d}\left\langle\hat{\sigma}_{z}\right\rangle}{\mathrm{d} t}=2 \Gamma^{-}\left\langle\hat{\sigma}_{-} \hat{\sigma}_{+}\right\rangle-2 \Gamma^{+}\left\langle\hat{\sigma}_{+} \hat{\sigma}_{-}\right\rangle
$$

where $\Gamma^{+}$and $\Gamma^{-}$are defined exactly in the same manner as in Sec. III. The above equation assumes that the field is initially in its ground state. Using relations $\hat{\sigma}_{z}=2 \hat{\sigma}_{+} \hat{\sigma}_{-}-1$ and $\hat{\sigma}_{-} \hat{\sigma}_{+}=1-\hat{\sigma}_{+} \hat{\sigma}_{-}$, it is found that the time evolution of the excited state probability, $\mathcal{P}_{e} \equiv\left\langle\hat{\sigma}_{+} \hat{\sigma}_{-}\right\rangle$, is determined by

$$
\frac{\mathrm{d} \mathcal{P}_{e}}{\mathrm{~d} t}=\Gamma^{-}-\mathcal{P}_{e}\left(\Gamma^{-}+\Gamma^{+}\right)
$$

The general solution of this differential equation is

$$
\mathcal{P}_{e}(t)=\frac{\Gamma^{-}}{\Gamma^{-}+\Gamma^{+}}\left(1-\mathrm{e}^{-\left(\Gamma^{-}+\Gamma^{+}\right) t}\right)+\mathcal{P}_{e}(0) \mathrm{e}^{-\left(\Gamma^{-}+\Gamma^{+}\right) t}
$$

where $\mathcal{P}_{e}(0)$ is the initial excited state's probability. In particular, this result shows that $-\left.\frac{d \mathcal{P}_{e} / d t}{\mathcal{P}_{e}}\right|_{t=0}=\Gamma^{+}-\Gamma^{-}$when $\mathcal{P}_{e}(0)=1 / 2$. Thus, $\Gamma_{\mathrm{gr}}=\Gamma^{+}-\Gamma^{-}$determines the initial time decay rate of the excited population in an ensemble of two-level atoms with an identical number of atoms in the excited and ground states at $t=0$. This shows that the total spontaneous emission rate $\Gamma_{\text {gr }}$ has a definite physical meaning also in the quantum problem.

As expected, Eq. (34) predicts that in the absence of relative motion (when $\Gamma^{-}=0$ ) the excited state probability decays exponentially to zero, so that the atom decays to the ground state $|g\rangle$ in a standard spontaneous emission process and in accordance with the results of section III. More interestingly, for velocities for which $\Gamma^{-}$differs significantly from zero, the probability of the excited state evolves towards a stationary value $\mathcal{P}_{e, \infty} \equiv \mathcal{P}_{e}(t=$ $\infty)=\Gamma^{-} /\left(\Gamma^{-}+\Gamma^{+}\right)$that depends on the relative strength of the decay rates $\Gamma^{+}, \Gamma^{-}$and that determines some sort of dynamic equilibrium of the two-level atom. Note that in the stationary regime $(t \rightarrow \infty)$ it is possible to write $\mathcal{P}_{e, \infty} \Gamma^{+}-\left(1-\mathcal{P}_{e, \infty}\right) \Gamma^{-}=0$, such that the number of transitions to the excited state induced by the relative motion $\left(1-\mathcal{P}_{e, \infty}\right) \Gamma^{-}$ 
equals the number of transitions to the ground state induced by the emission processes $\mathcal{P}_{e, \infty} \Gamma^{+}$.

The picture that emerges from this result is that the limit $t \rightarrow \infty$ may have a dynamical character, such that energy is continuously extracted from the kinetic degrees of freedom and emitted as light. Indeed, the fact that $\mathcal{P}_{e, \infty}$ is nonzero proves that in the Schrödinger picture the atomic wave function is a mixed state for large $t$, and the expectation of the dipole moment is nonzero for any mixed state $\left(\left\langle\hat{\sigma}_{-}\right\rangle \neq 0\right)$.

Notably, when the classical decay rate $\Gamma_{\mathrm{cl}}=\Gamma_{\mathrm{gr}}=\Gamma^{+}-\Gamma^{-}$is negative, i.e., the transitions with negative Doppler shifted frequency dominate over the emission processes with positive Doppler shifted frequency, then $\mathcal{P}_{e, \infty}>1 / 2$. In this situation, a population inversion takes place, independently of the initial atomic state.

Importantly, a two-level atom is a system with an intrinsic saturation mechanism, because its energy is bounded from above. Moreover, the dipole moment amplitude is also bounded, and reaches the maximum for mixed-states with $\mathcal{P}_{e, \infty}=1 / 2$, i.e., at the onset of the classical instability. Hence, different from the classical case, in the quantum problem the dipole moment cannot grow exponentially, and the maximum oscillation amplitude is reached for $\mathcal{P}_{e, \infty}=1 / 2$. This suggests that classical situations leading to strong instabilities should correspond in the quantum case to $\mathcal{P}_{e, \infty}$ marginally larger than $1 / 2$. In section VI it will be shown with a numerical example that this is indeed the case. The fact that $\mathcal{P}_{e, \infty}>1 / 2$ suggests that if a ladder of energy levels would be accessible then the dipole oscillations would grow exponentially in time, similar to the classical problem.

In summary, the picture that emerges from our quantum optics analysis is that the twolevel atom evolves towards a dynamical equilibrium state, such that the number of transitions in the ascending direction equals the number of transitions in the descending direction.

\section{Friction force}

As discussed in section III, the transitions associated with oscillators with negative frequencies must be accompanied by a conversion of kinetic energy into light. The optical friction force responsible for this exchange of energy can be calculated in the frame co-moving with the two-level atom using $\mathcal{F}_{x}=\mathbf{p}_{e} \cdot \partial_{x^{\prime}} \mathbf{E}^{\prime}=\mathbf{p} \cdot \partial_{x^{\prime}} \mathbf{F}^{\prime}$, where the partial derivative acts over the spatial coordinate along the direction of motion [56]. Promoting all the relevant 
physical quantities to operators and using normal ordering, it is follows that the quantum expectation of the force is:

$$
\left\langle\mathcal{F}_{x}\left(\mathbf{r}_{0}^{\prime}\right)\right\rangle=2 \operatorname{Re}\left\{\left\langle\hat{\mathbf{p}} \cdot \partial_{x^{\prime}} \hat{\mathbf{F}}^{\prime-}\left(\mathbf{r}_{0}^{\prime}\right)\right\rangle\right\}
$$

Using Eqs. (29)-(30) and assuming that at initial time the field is in the vacuum state it follows that in the non-relativistic limit the force is given by:

$$
\begin{aligned}
\left\langle\hat{\mathcal{F}}_{x}\left(\mathbf{r}_{0}^{\prime}\right)\right\rangle= & \sum_{\omega_{n \mathbf{k}}>0} \operatorname{Re}\left\{i \pi \omega_{n \mathbf{k}} \tilde{\boldsymbol{\gamma}}_{e g}^{*} \cdot\left[\partial_{x^{\prime}} \mathbf{F}_{n \mathbf{k}}\left(\mathbf{r}_{0}^{\prime}\right) \otimes \mathbf{F}_{n \mathbf{k}}^{*}\left(\mathbf{r}_{0}^{\prime}\right)\right] \cdot \tilde{\gamma}_{e g} \delta\left(\omega_{0}-\omega_{n \mathbf{k}}^{\prime}\right)\left\langle\hat{\sigma}_{+} \hat{\sigma}_{-}\right\rangle\right\} \\
& +\sum_{\omega_{n \mathbf{k}}>0} \operatorname{Re}\left\{i \pi \omega_{n \mathbf{k}} \tilde{\gamma}_{e g} \cdot\left[\partial_{x^{\prime}} \mathbf{F}_{n \mathbf{k}}\left(\mathbf{r}_{0}^{\prime}\right) \otimes \mathbf{F}_{n \mathbf{k}}^{*}\left(\mathbf{r}_{0}^{\prime}\right)\right] \cdot \tilde{\boldsymbol{\gamma}}_{e g}^{*} \delta\left(\omega_{0}+\omega_{n \mathbf{k}}^{\prime}\right)\left\langle\hat{\sigma}_{-} \hat{\sigma}_{+}\right\rangle\right\}
\end{aligned}
$$

The system is invariant to translations along the direction of motion and thus for the mode $n \mathbf{k}$ one has $\partial_{x^{\prime}}=i k_{x}$, being $k_{x}$ the $x$-component of the wave vector. Hence, writing the expectation of the atomic operators in terms of $\mathcal{P}_{e}$, one finally finds that the friction force is

$$
\begin{aligned}
\left\langle\hat{\mathcal{F}}_{x}\left(\mathbf{r}_{0}^{\prime}\right)\right\rangle= & -\mathcal{P}_{e} \sum_{\omega_{n \mathbf{k}}>0} \operatorname{Re}\left\{\pi k_{x} \omega_{n \mathbf{k}} \tilde{\gamma}_{e g}^{*} \cdot\left[\mathbf{F}_{n \mathbf{k}}\left(\mathbf{r}_{0}^{\prime}\right) \otimes \mathbf{F}_{n \mathbf{k}}^{*}\left(\mathbf{r}_{0}^{\prime}\right)\right] \cdot \tilde{\gamma}_{e g} \delta\left(\omega_{0}-\omega_{n \mathbf{k}}^{\prime}\right)\right\} \\
& -\left(1-\mathcal{P}_{e}\right) \sum_{\omega_{n \mathbf{k}}>0} \operatorname{Re}\left\{\pi k_{x} \omega_{n \mathbf{k}} \tilde{\gamma}_{e g} \cdot\left[\mathbf{F}_{n \mathbf{k}}\left(\mathbf{r}_{0}^{\prime}\right) \otimes \mathbf{F}_{n \mathbf{k}}^{*}\left(\mathbf{r}_{0}^{\prime}\right)\right] \cdot \tilde{\gamma}_{e g}^{*} \delta\left(\omega_{0}+\omega_{n \mathbf{k}}^{\prime}\right)\right\} .
\end{aligned}
$$

It is demonstrated in the next section that in the quasi-static limit the friction force can be directly written in terms of the transition rates $\Gamma^{-}$and $\Gamma^{+}$.

\section{THE QUASI-STATIC LIMIT}

\section{A. Analytical development}

To illustrate the concepts developed throughout this paper and link them to the classical results of Ref. [38], next we derive explicit formulas for the spontaneous emission rates and friction force using a quasi-static approximation for the quantized electromagnetic fields.

In a quasi-static approximation the fields are purely electric $\mathbf{F}_{n \mathbf{k}} \approx\left(\begin{array}{ll}\mathbf{E}_{\mathbf{k}} & \mathbf{0}\end{array}\right)^{T}$ [57], and the electric field $\mathbf{E}_{\mathbf{k}}=-\nabla \phi_{\mathbf{k}}$ is written in terms of an electric potential that satisfies $\nabla \cdot\left(\varepsilon(\omega, z) \nabla \phi_{\mathbf{k}}\right)=0$. Assuming that the plasmonic slab is located in $z<0$ region (so that the metal-air boundary is at $z=0$ ), it follows that the electric potential must be of the form

$$
\phi_{\mathbf{k}}=A_{\mathbf{k}} e^{i \mathbf{k}_{||} \cdot \mathbf{r}} e^{-k_{||}|z|},
$$


where $\mathbf{k}_{\|}=k_{x} \hat{\mathbf{x}}+k_{y} \hat{\mathbf{y}}$ is the wave vector (here, we use the subscript $\|$ to highlight that the wave vector is parallel to the interface) and $A_{\mathbf{k}}$ is a normalization constant. Moreover, the relevant eigenfrequency is $\omega_{\mathbf{k}}=\omega_{\mathrm{sp}}$ where $\omega_{\mathrm{sp}}$ corresponds to the surface plasmon resonance $\varepsilon\left(\omega_{s p}\right)=-\varepsilon_{0}$.

To determine the value of $A_{\mathbf{k}}$, we use the normalization condition (6) that gives

$$
\left|A_{\mathbf{k}}\right|^{2} \int d^{3} \mathbf{r} k_{\|}^{2} \mathrm{e}^{-2 k_{\|}|z|} \frac{\partial[\omega \varepsilon(\omega, z)]}{\partial \omega}=1
$$

Considering that the permittivity of the plasmonic slab is modeled by a lossless Drude model, $\varepsilon=\varepsilon_{0}\left(1-2 \omega_{\mathrm{sp}}^{2} / \omega^{2}\right)$, one has

$$
\frac{\partial[\omega \varepsilon(\omega, z)]}{\partial \omega} \stackrel{\omega=\omega_{\mathrm{sp}}}{\longrightarrow}\left\{\begin{array}{cc}
\varepsilon_{0}, & z>0 \\
3 \varepsilon_{0}, & z<0
\end{array}\right.
$$

and hence (39) gives that

$$
\left|A_{\mathbf{k}}\right|=\sqrt{\frac{1}{2 k_{\|} \varepsilon_{0} S_{0}}}
$$

where $S_{0}$ is surface area of the plasmonic slab.

Substituting the quasi-static electric field into Eqs. (11), (12), (21) and (37), it is now straightforward to determine the spontaneous emission rates, the interaction's dyadic and the friction force. For simplicity, in what follows it is supposed that the electric dipole is oriented along the $z$-direction so that $\gamma_{e g}=\gamma_{e g} \hat{\mathbf{z}}$. In this case the transition rate to the ground state (11) simplifies to:

$$
\Gamma^{+}=\sum_{\mathbf{k}_{\|}} \frac{\pi \omega_{\mathrm{sp}}}{\hbar} \frac{k_{\|}}{2 \varepsilon_{0} S_{0}}\left|\gamma_{e g}\right|^{2} \mathrm{e}^{-2 k_{\|} d} \delta\left(\omega_{\mathrm{sp}}+k_{x} v-\omega_{0}\right),
$$

where $d$ is the distance between the two-level atom and the plasmonic slab. The sum over $\mathbf{k}_{\|}$ can be transformed into an integral by substituting $\frac{1}{S_{0}} \sum_{\mathbf{k}_{\|}} \rightarrow \frac{1}{(2 \pi)^{2}} \iint d k_{x} d k_{y}$. This gives:

$$
\Gamma^{+}=\frac{2\left|\gamma_{e g}\right|^{2}}{\varepsilon_{0} \hbar} \frac{1}{d^{3}} G\left(\frac{\omega_{0} d}{|v|}, \frac{\omega_{\mathrm{sp}} d}{|v|}\right)
$$

where we introduced the function

$$
G(a, b)=\frac{b}{8 \pi} \int_{0}^{\infty} \sqrt{u^{2}+(a-b)^{2}} \mathrm{e}^{-2 \sqrt{u^{2}+(a-b)^{2}}} \mathrm{~d} u .
$$


Similarly, using Eq. (12) it is readily verified that the rate of transitions to the excited state is

$$
\Gamma^{-}=\frac{2\left|\gamma_{e g}\right|^{2}}{\varepsilon_{0} \hbar} \frac{1}{d^{3}} G\left(\frac{-\omega_{0} d}{|v|}, \frac{\omega_{\mathrm{sp}} d}{|v|}\right) .
$$

Now, using the fact that $\Gamma_{\mathrm{cl}}=\Gamma^{+}-\Gamma^{-}$and Eq. (18) one sees that the $z z$ component of the interaction dyadic satisfies:

$$
\operatorname{Im}\left\{C_{\mathrm{int}, z z}\right\}=\frac{1}{d^{3}}\left[G\left(\frac{\omega_{0} d}{|v|}, \frac{\omega_{\mathrm{sp}} d}{|v|}\right)-G\left(\frac{-\omega_{0} d}{|v|}, \frac{\omega_{\mathrm{sp}} d}{|v|}\right)\right]
$$

This result is exactly coincident with Eq. (15) of Ref. [38], which was derived using a totally different approach. This agreement provides an independent check of the theoretical concepts introduced in this article. It should be noted that in the quasi-static approximation, the free space's contribution to the interaction constant is $\operatorname{lost}\left(\operatorname{Im}\left\{\underline{C}_{\text {tot }}\right\}=\operatorname{Im}\left\{\underline{C}_{\text {int }}\right\}\right)$. However, as demonstrated in [38], this contribution is tiny as compared to the imaginary part of $C_{\text {int, } z z}$ and hence can be safely neglected.

On the other hand, it can be shown that within the same approximations the friction force acting on the two-level atom (37) simplifies to

$$
\left\langle\hat{\mathcal{F}}_{x}\right\rangle=-\mathcal{P}_{e} \Gamma^{+} \hbar \frac{\omega_{0}-\omega_{\mathrm{sp}}}{v}+\left(1-\mathcal{P}_{e}\right) \Gamma^{-} \hbar \frac{\omega_{0}+\omega_{\mathrm{sp}}}{v}
$$

and in the stationary state $(t \rightarrow \infty)$ it further reduces to

$$
\left\langle\hat{\mathcal{F}}_{x, \infty}\right\rangle=\frac{2 \hbar \omega_{\mathrm{sp}}}{v} \frac{\Gamma^{+} \Gamma^{-}}{\Gamma^{+}+\Gamma^{-}} .
$$

Note that the signs of the friction force and atom velocity are opposite because the velocity of the atom is $-v$ in the reference frame of the metal slab $(v$ represents the relative velocity of the metal with respect to the atom). Interestingly, the above expression shows that whenever the rate of ascending transitions $\Gamma^{-}$is nonzero then the friction force will also be nonzero, even if the corresponding classical problem is stable (i.e. $\Gamma^{+}>\Gamma^{-}$). This situation contrasts with the case of sliding lossless slabs, where a friction force implies an unstable response [13]. The difference is that in the latter problem the "emitter" is infinitely extended in space and hence without absorption the emitted energy will necessarily build up, leading to an exponential growth. On the other hand, for a moving atom the emitted light can be radiated away and thus it does not necessarily increase the stored energy in the vicinity of the atom. 
Similarly, the energy emitted per unit of time is given by $\left\langle\hat{\mathcal{F}}_{x, \infty}\right\rangle v=2 \hbar \omega_{\mathrm{sp}} \mathcal{P}_{e, \infty} \Gamma^{+}$(in agreement with the transition rates evaluated in section VB; the leading factor of "2" can be understood noting that both the ascending and descending transitions emit a plasmon). Hence, the emitted power saturates at some value rather than growing exponentially as in the classical case.

\section{B. Numerical study}

We are now ready to study how the dynamic equilibrium state $\mathcal{P}_{e, \infty}=\Gamma^{-} /\left(\Gamma^{-}+\Gamma^{+}\right)$ induced by the interaction with the SPPs varies with the relative velocity. The excited state probability in the limit $t \rightarrow \infty$ depends only on the normalized parameters $\omega_{0} d /|v|$ and $\omega_{\text {sp }} d /|v|$. A density plot of $\mathcal{P}_{e, \infty}$ as a function of the two normalized variables is represented in Fig. 2 (a) in a parametric range near to the maximum of the function. As seen, the maximum value of $\mathcal{P}_{e, \infty}$ occurs exactly for $\omega_{0}=\omega_{\text {sp }}$, i.e., when the atomic transition frequency is coincident with the SPP resonance. Furthermore, as expected, for small velocities (which correspond to large values of the normalized parameters) the value of $\mathcal{P}_{e, \infty}$ is negligible. A detailed variation of $\mathcal{P}_{e, \infty}$ in the interesting case $\omega_{0}=\omega_{\mathrm{sp}}$ is depicted in Fig. 2 (b). The optimal value that maximizes $\mathcal{P}_{e, \infty}$ is $\omega_{\text {sp }} d / v=0.148$ and corresponds to the optimal velocity $v \approx 6.72 \omega_{\mathrm{sp}} d$.

Notably, there is a range of parameters for which $\mathcal{P}_{e, \infty}>1 / 2$ implying a negative spontaneous emission $\Gamma_{\mathrm{gr}}<0$. Moreover, consistent with the discussion of Sec. V A, in such a regime the maximum value (about 0.515 ) reached by $\mathcal{P}_{e, \infty}$ is only marginally larger than $1 / 2$. This ensures that the dipole oscillation amplitude in the dynamical equilibrium has the largest possible value, when the associated classical problem is characterized by an unstable response. Indeed, whereas in the classical case [38] the dipole moment amplitude may reach arbitrarily large values (in the linear regime), in a two-level atom it saturates for mixed states with $\mathcal{P}_{e} \approx 1 / 2$.

It is interesting to look at an example with a realistic material with more detail. Here, as in Ref. [38] we consider that the metal is silver, which is assumed to be modeled by a Drude model with a plasma frequency $\omega_{\mathrm{sp}} /(2 \pi)=646 \mathrm{THz}$ [58]. The effect of metallic loss is not included in our calculation, but as shown in Ref. [38] it does not change the qualitative picture, and only acts to reduce the strength of the negative spontaneous emission rate. 

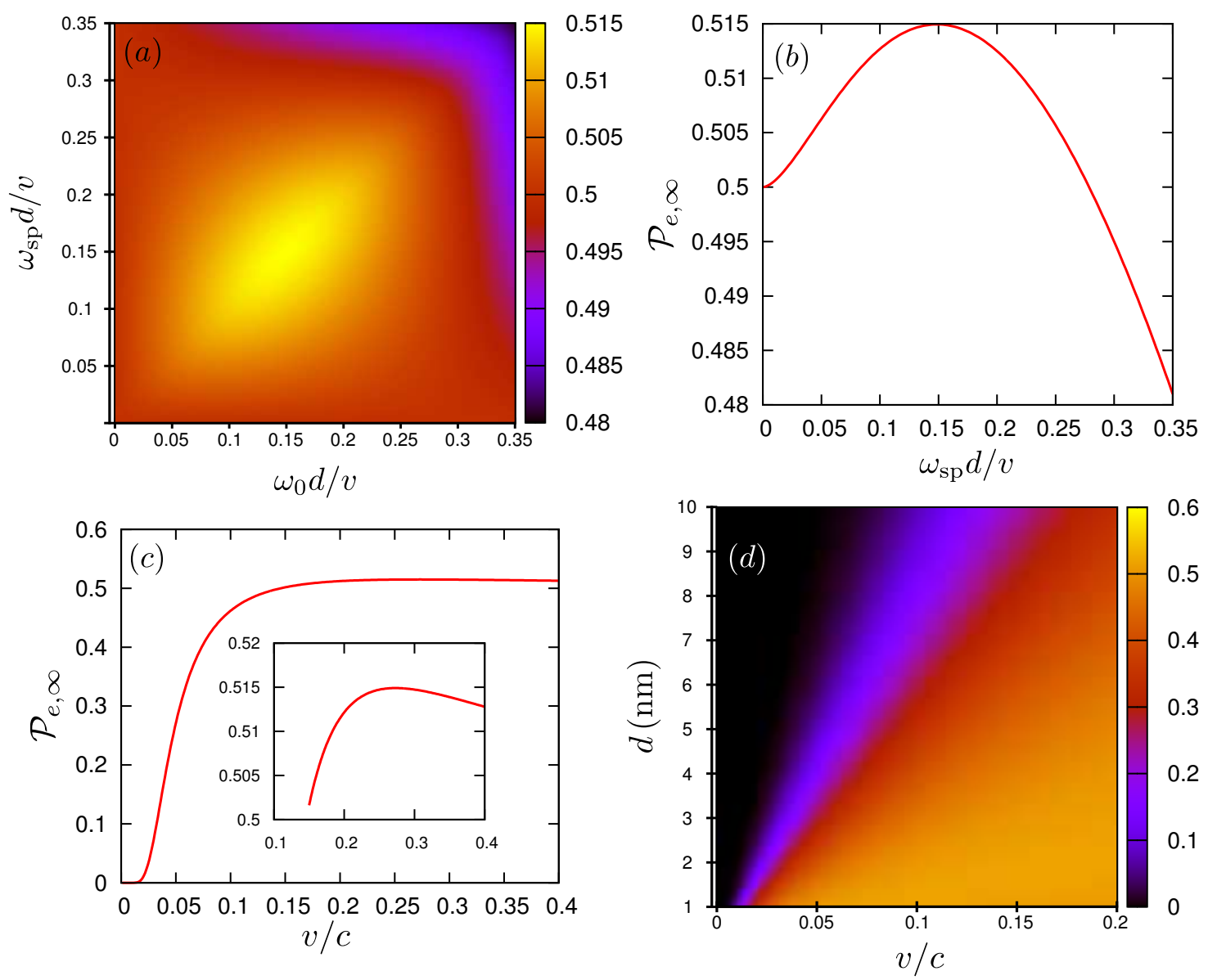

FIG. 2. Plot of the probability $\mathcal{P}_{e, \infty}$ in different situations: (a) Density plot of $\mathcal{P}_{e, \infty}$ as a function of the dimensionless parameters $\omega_{0} d / v$ and $\omega_{\mathrm{sp}} d / v$. (b) $\mathcal{P}_{e, \infty}$ as a function of $\omega_{\mathrm{sp}} d / v$ for $\omega_{0}=\omega_{\mathrm{sp}}$. (c) $\mathcal{P}_{e, \infty}$ as a function of the velocity $v$ for a two-level atom located at a distance $d=3 \mathrm{~nm}$ from a lossless silver slab with $\omega_{\mathrm{sp}} /(2 \pi)=646 \mathrm{THz}$ and an atomic transition frequency $\omega_{0}=\omega_{\mathrm{sp}}$. The inset shows a zoom of the same curve near $\mathcal{P}_{e, \infty}=1 / 2$. (d) Density plot of $\mathcal{P}_{e, \infty}$ as a function of the velocity and of the distance, for $\omega_{\mathrm{sp}} /(2 \pi)=646 \mathrm{THz}$ and $\omega_{0}=\omega_{\mathrm{sp}}$.

Figure 2 (c) shows the variation of $\mathcal{P}_{e, \infty}$ with the relative velocity for a distance $d=3 \mathrm{~nm}$ between the two-level atom and the silver slab, and an atomic transition frequency $\omega_{0}=\omega_{\mathrm{sp}}$. As seen for low velocities (typically $v<0.05 c$ ), $\mathcal{P}_{e, \infty}$ remains near zero meaning that the processes involving negative Doppler shifted frequency are negligible in agreement with the conclusions of Secs. III and V. Crucially, for higher velocities the situation changes, and as we enter in the range of parameters shown in Fig. 2 (a)-(b), the excited state probability in the dynamical equilibrium reaches a value around $50 \%$. In addition, as can be seen in the inset, the probability reaches a maximum for the velocity $v \approx 0.273 c$. 
To have a clear idea of the range of parameters needed for a negative spontaneous emission we represent in Fig. 2 (d) a density plot of $\mathcal{P}_{e, \infty}$ as a function of the velocity and of the distance, for the same scenario as in the previous example. Obviously, increasing the distance between the atom and the slab weakens the interaction, and in particular the effect of the quantum oscillators with negative Doppler shifted frequencies. As a consequence, the threshold velocity to obtain a population inversion is higher. We note that the effects of timeretardation and relativistic corrections are not expected to change the general conclusions of the article, as it was shown in Ref. [38] that they generally correspond to small corrections in the classical case.

Next, we characterize the quantum friction force. Figure 3 shows the equilibrium friction force $\left\langle\hat{\mathcal{F}}_{x, \infty}\right\rangle$ as well as the power radiated by the system $\left\langle\hat{\mathcal{F}}_{x, \infty}\right\rangle v$ as a function of the normalized parameters $\omega_{0} d /|v|$ and $\omega_{\mathrm{sp}} d /|v|$. As expected, the force and the emitted power
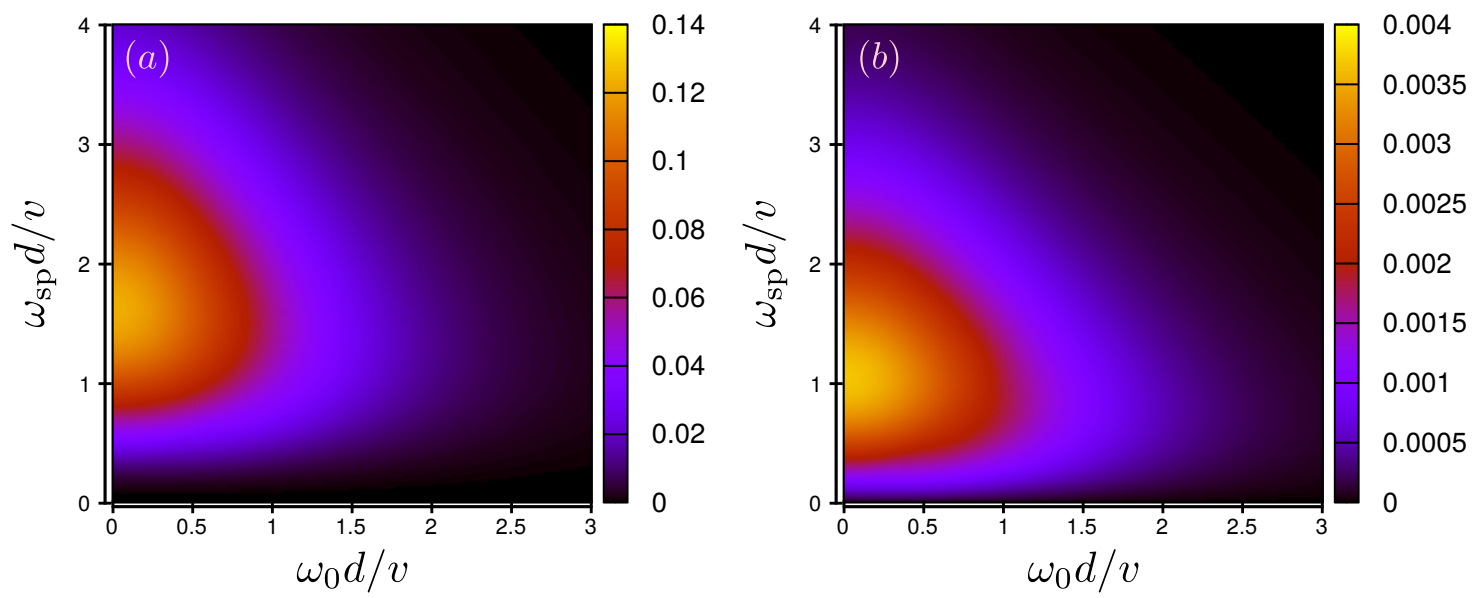

FIG. 3. Plot of the expectation value of (a) the normalized friction force $\left\langle\mathcal{F}_{x, \infty}\right\rangle \cdot \frac{4 \pi \varepsilon_{0} d^{4}}{\left|\gamma_{e g}\right|^{2}}$ and (b) the normalized radiated power $\langle\mathcal{P}\rangle \cdot \frac{4 \pi \varepsilon_{0} d^{3}}{\omega_{\mathrm{sp}}\left|\gamma_{e g}\right|^{2}}$ as a function of the dimensionless parameters $\omega_{0} d / v$ and $\omega_{\mathrm{sp}} d / v$.

are significant only for sufficiently high velocities and small distances. Comparing the plot of $\mathcal{P}_{e, \infty}$ (Fig. 2 (a)) with the plots in Fig. 3, it is somewhat surprising that the maxima of $\mathcal{P}_{e, \infty}$ and $\left\langle\mathcal{F}_{x, \infty}\right\rangle$ occur in rather different regions of the parameter space. Indeed, the maxima of the friction force and power occur for $\omega_{0} \ll \omega_{\text {sp }}$, whereas the maximum of $\mathcal{P}_{e, \infty}$ occurs for $\omega_{0}=\omega_{\text {sp }}$. Yet, it is possible to see from equations (43) and (45) that a case with $\omega_{0} \ll \omega_{\text {sp }}$ corresponds to $\Gamma^{+} \approx \Gamma^{-}$and hence to $\mathcal{P}_{e, \infty} \approx 1 / 2$. Thus, even though the maximum of friction force does not fall within the region wherein $\mathcal{P}_{e, \infty}$ exceeds $1 / 2$, the 
value of $\mathcal{P}_{e, \infty}$ is actually only marginally smaller than the maximum. For example, at the maximum of the friction force $\left(w_{0} d / v=0^{-}\right.$and $\left.w_{\mathrm{sp}} d / v \approx 1.62\right)$ one has $\mathcal{P}_{e, \infty}=1 / 2+0^{-}$. The key point that shows there is no lack of consistency is that if the atom had an infinite number of energy levels the quantum friction would likely grow exponentially with time, and hence in such a case the peak friction force would necessarily fall within the region wherein the total spontaneous emission rate is negative.

We verified that within our model the friction force becomes exponentially small in the $v \rightarrow 0$ limit. This property is consistent with a result obtained by Pendry for lossless plasmonic slabs in a shear motion [25]. In contrast, for dissipative materials with a nonzero conductivity in the static limit, the friction force exhibits a power law dependence on the velocity $[14,37,56]$. Indeed, it is important to highlight that in our problem the friction force is exclusively due to the conversion of kinetic energy into electromagnetic radiation (plas-

mons) because the system is lossless. This process is particularly efficient when $\omega_{\text {sp }} d / v \sim 1$ (see Fig. 3). On the other hand, lossy materials provide additional channels for dissipation, namely enable the conversion of kinetic energy into heat, and this explains why the friction force is stronger in the $v \rightarrow 0$ limit in dissipative systems.

\section{CONCLUSION}

It was demonstrated that the rate of spontaneous emission by a moving two-level atom interacting with the near-field of a plasmonic slab is, in the most general case, determined by two concurrent processes: the conservative ones involving only positive Doppler shifted frequencies and the non-conservatives involving only negative Doppler shifted frequencies. The transitions associated with negative Doppler shifted frequencies are due to the conversion of kinetic energy into light [16].

It was proven that when the non-conservative processes are dominant, the system is characterized by a negative spontaneous emission meaning that the transition rate to the excited state exceeds the transition rate to the ground state. In this regime, the corresponding classical problem is characterized by an unstable response. Different from the classical situation, in the quantum case the dipole moment oscillations are intrinsically bounded. It was shown with a numerical example that the unstable regime corresponds in the quantum system to a saturation of the dipole moment amplitude. 
The links between the quantum theory presented here and the classical theory of Ref. [38] have been emphasized. In particular, it was shown that the classical decay rate is exactly coincident with the quantum spontaneous emission rate. Using a quasi-static approximation for the electromagnetic field, the typical range of distances and velocities to observe negative spontaneous emission and an inversion of population have been detailed. Additionally, we studied the phenomenon of quantum friction. Different from its classical counterpart [38], the quantum friction effect in the considered system has no velocity threshold and in particular may occur even when the total spontaneous emission rate remains positive, i.e., when the classical system is stable. Moreover, the regime wherein the friction force is maximal does not overlap with the regime wherein the spontaneous emission rate is negative. Thus, even though the stability threshold of the classical problem is coincident with the threshold of negative spontaneous emission, the physics of the classical and quantum systems is generally rather different mainly due to the unique energy spectrum of the two-level atom which prevents the development of instabilities in the quantum case.

\section{ACKNOWLEDGMENTS}

This work was partially funded by Fundação para Ciência e a Tecnologia under project PTDC/EEI-TEL/4543/2014.

\section{Appendix A: The field radiated by a moving electric dipole}

In the following, we calculate the electromagnetic field radiated by a moving electric dipole in the reference frame co-moving with the plasmonic slab. The electromagnetic field satisfies the Maxwell's equations

$$
\hat{N} \mathbf{F}=i \frac{\partial}{\partial t} \mathbf{M} \cdot \mathbf{F}+i \mathbf{J}
$$

where $\mathbf{J}$ is a six-vector representing the current density due to the moving source. For a moving dipole it is of the form:

$$
\mathbf{J}(\mathbf{r}, t)=\frac{d}{d t}\left[\delta\left(\mathbf{r}-\mathbf{r}_{0}(t)\right) \mathbf{p}(t)\right]
$$

where $\mathbf{r}_{0}(t)=\mathbf{r}_{0}^{\prime}-\mathbf{v} t$ are the coordinates of the two-level atom in the reference frame of the plasmonic slab, and $\mathbf{p}=\left(\begin{array}{ll}\mathbf{p}_{e} & \mathbf{p}_{m}\end{array}\right)^{T}$ is the generalized dipole moment, such that $\mathbf{p}_{e}$ 
and $\mathbf{p}_{m}$ represent the electric and magnetic dipole moments (the magnetic dipole moment is included here only for the sake of generality). The radiated field can be written in terms of the temporal Green-function as

$$
\mathbf{F}(\mathbf{r}, t)=\iint \underline{G}\left(\mathbf{r}, \mathbf{r}_{1}, t-t_{1}\right) \cdot \mathbf{J}\left(\mathbf{r}_{1}, t_{1}\right) d \mathbf{r}_{1} d t_{1},
$$

where $\underline{G}\left(\mathbf{r}, \mathbf{r}_{1}, t\right)$ is the solution of

$$
\left(\hat{N}-i \frac{\partial}{\partial t} \mathbf{M}\right) \cdot \underline{G}\left(\mathbf{r}, \mathbf{r}_{1}, t\right)=i \delta\left(\mathbf{r}-\mathbf{r}_{1}\right) \delta(t) \mathbf{1}_{6 \times 6}
$$

that satisfies the causality condition $\underline{G}\left(\mathbf{r}, \mathbf{r}_{1}, t\right)=0$ for $t<0$. Assuming that the time dependence of the dipole moment is of the form $\mathbf{p}(t)=\mathbf{p} e^{-i \omega t}$ it is readily found that:

$$
\mathbf{F}(\mathbf{r}, t)=\frac{d}{d t}\left[\int \underline{G}\left(\mathbf{r}, \mathbf{r}_{0}\left(t_{1}\right), t-t_{1}\right) \cdot \mathbf{p} e^{-i \omega t_{1}} d t_{1}\right] .
$$

In Appendix B, it is shown that the Green's function in the spectral domain can be expanded in terms of the electromagnetic eigenmodes $\mathbf{F}_{n \mathbf{k}}$ as in Eq. (B9), so that in the time domain:

$$
\underline{G}\left(\mathbf{r}, \mathbf{r}_{1}, t\right)=-u(t) \frac{1}{2} \sum_{n \mathbf{k}} e^{-i \omega_{n \mathbf{k}} t} \mathbf{F}_{n \mathbf{k}}(\mathbf{r}) \otimes \mathbf{F}_{n \mathbf{k}}^{*}\left(\mathbf{r}_{1}\right),
$$

where $\otimes$ denotes the tensor product of two six-vectors, and $u(t)$ is the Heaviside step function. The eigenmodes are normalized as in Eq. (6), and the sum includes modes with positive, negative and zero frequencies. Substituting this result into Eq. (A5) one obtains:

$$
\mathbf{F}(\mathbf{r}, t)=\sum_{n \mathbf{k}} \frac{\omega-\mathbf{k} \cdot \mathbf{v}}{2\left(\omega_{n \mathbf{k}}^{\prime}-\omega\right)} \mathbf{F}_{n \mathbf{k}}(\mathbf{r}+\mathbf{v} t) \otimes \mathbf{F}_{n \mathbf{k}}^{*}\left(\mathbf{r}_{0}^{\prime}\right) \cdot \mathbf{p} \mathrm{e}^{-i \omega t},
$$

where $\omega_{n \mathbf{k}}^{\prime}=\omega_{n \mathbf{k}}+\mathbf{k} \cdot \mathbf{v}$ is the Doppler shifted frequency. Using the following completeness relation (which can be derived using ideas analogous to those described in Appendix B; $\left.\mathbf{M}_{\infty} \equiv \lim _{\omega \rightarrow \infty} \mathbf{M}(\mathbf{r}, \omega)\right)$

$$
\sum_{n \mathbf{k}} \frac{1}{2} \mathbf{F}_{n \mathbf{k}}(\mathbf{r}) \otimes \mathbf{F}_{n \mathbf{k}}^{*}\left(\mathbf{r}_{1}\right)=\mathbf{M}_{\infty}^{-1} \delta\left(\mathbf{r}-\mathbf{r}_{1}\right),
$$

and supposing that the dipole is in the free-space region (with material matrix $\mathbf{M}_{0}$ ), it is found that:

$$
\mathbf{F}=e^{-i \omega t} \sum_{n \mathbf{k}} \frac{1}{2} \frac{\omega_{n \mathbf{k}}}{\left(\omega_{n \mathbf{k}}^{\prime}-\omega\right)} \mathbf{F}_{n \mathbf{k}}(\mathbf{r}+\mathbf{v} t) \mathbf{F}_{n \mathbf{k}}^{*}\left(\mathbf{r}_{0}^{\prime}\right) \cdot \mathbf{p}-e^{-i \omega t} \mathbf{M}_{0}^{-1} \cdot \mathbf{p} \delta\left(\mathbf{r}+\mathbf{v} t-\mathbf{r}_{0}^{\prime}\right)
$$


The reality of the electromagnetic field implies that the above summation can be restricted to eigenmodes with positive frequencies as follows:

$$
\begin{aligned}
\mathbf{F}(\mathbf{r}, t)= & \sum_{\omega_{n \mathbf{k}}>0} \frac{\omega_{n \mathbf{k}}}{2}\left(\frac{1}{\omega_{n \mathbf{k}}^{\prime}-\omega} \mathbf{F}_{n \mathbf{k}}(\mathbf{r}+\mathbf{v} t) \otimes \mathbf{F}_{n \mathbf{k}}^{*}\left(\mathbf{r}_{0}^{\prime}\right)+\frac{1}{\omega_{n \mathbf{k}}^{\prime}+\omega} \mathbf{F}_{n \mathbf{k}}^{*}(\mathbf{r}+\mathbf{v} t) \otimes \mathbf{F}_{n \mathbf{k}}\left(\mathbf{r}_{0}^{\prime}\right)\right) \cdot \mathbf{p} \mathrm{e}^{-i \omega t} \\
& -\delta\left(\mathbf{r}+\mathbf{v} t-\mathbf{r}_{0}^{\prime}\right) \mathbf{M}_{0}^{-1} \cdot \mathbf{p} \mathrm{e}^{-i \omega t}
\end{aligned}
$$

Equation (A10) gives the exact solution for the fields radiated by the moving dipole in the

reference frame of the plasmonic slab. Similar to Sec. II, to obtain the corresponding fields in the frame co-moving with the dipole we use simply the non-relativistic approximation $\mathbf{F}^{\prime}\left(\mathbf{r}^{\prime}, t^{\prime}\right) \approx \mathbf{F}\left(\mathbf{r}^{\prime}-\mathbf{v} t, t\right)$. Note that $\mathbf{p}^{\prime}\left(t^{\prime}\right) \approx \mathbf{p}(t)$ within the same non-relativistic approximation and hence the frequency of oscillation of the dipole does not need to be transformed. Therefore, one concludes that

$$
\begin{aligned}
\mathbf{F}^{\prime}\left(\mathbf{r}^{\prime}, t\right) \approx & \sum_{\omega_{n \mathbf{k}}>0} \frac{\omega_{n \mathbf{k}}}{2}\left(\frac{1}{\omega_{n \mathbf{k}}^{\prime}-\omega} \mathbf{F}_{n \mathbf{k}}\left(\mathbf{r}^{\prime}\right) \otimes \mathbf{F}_{n \mathbf{k}}^{*}\left(\mathbf{r}_{0}^{\prime}\right)+\frac{1}{\omega_{n \mathbf{k}}^{\prime}+\omega} \mathbf{F}_{n \mathbf{k}}^{*}\left(\mathbf{r}^{\prime}\right) \otimes \mathbf{F}_{n \mathbf{k}}\left(\mathbf{r}_{0}^{\prime}\right)\right) \cdot \mathbf{p e}^{-i \omega t} \\
& -\delta\left(\mathbf{r}^{\prime}-\mathbf{r}_{0}^{\prime}\right) \mathbf{M}_{0}^{-1} \cdot \mathbf{p} \mathrm{e}^{-i \omega t}
\end{aligned}
$$

\section{Appendix B: Eigenmode expansion of the Green's function in the spectral domain}

In this appendix, we derive an explicit formula for the Green's function in the frequency domain $\underline{G}=\underline{G}\left(\mathbf{r}, \mathbf{r}_{1}, \omega\right)$, which from Eq. (A4) satisfies:

$$
(\hat{N}-\omega \mathbf{M}) \cdot \underline{G}\left(\mathbf{r}, \mathbf{r}_{1}, \omega\right)=i \delta\left(\mathbf{r}-\mathbf{r}_{1}\right) \mathbf{1}_{6 \times 6} .
$$

It is well known that the Green's function can be expanded as a sum of eigenvectors when all the involved materials are dispersionless [4, 59]. In contrast, in presence of material dispersion the application of the spectral theorem is not direct because the relevant differential operators are not Hermitian. To circumvent this problem, we use the ideas of Ref. [46, 48] to describe the material dispersion in terms of additional variables. Specifically, to obtain an Hermitian eigenvalue problem, the system (A1) may be transformed into the equivalent generalized problem [46]

$$
\hat{L} \cdot \mathbf{Q}=i \cdot \frac{\partial}{\partial t} \mathbf{M}_{g} \cdot \mathbf{Q}+i \mathbf{J}_{g}
$$

where $\hat{L}$ and $\mathbf{M}_{g}$ are (frequency independent) operators that can be constructed as explained in Ref. [46] and $\mathbf{Q}=\left(\begin{array}{lllll}\mathbf{F} & \mathbf{Q}_{1} & \mathbf{Q}_{2} & \ldots\end{array}\right)^{T}$ is a generalized state vector whose first sixcomponents determine the electromagnetic field $\mathbf{F}$. This transformation is possible provided 
all the materials are lossless. The number of additional variables $\mathbf{Q}_{i}$ 's is determined by the number of poles of $\mathbf{M}(\omega)$. In general both $\hat{L}$ and $\mathbf{M}_{g}$ are space dependent. The generalized excitation vector is of the form $\mathbf{J}_{g}=\left(\begin{array}{llll}\mathbf{J} & \mathbf{0} & \mathbf{0} & \ldots\end{array}\right)^{T}[46,48]$.

The eigenmodes of the generalized system (B2) satisfy:

$$
\mathbf{M}_{g}^{-1} \cdot \hat{L} \mathbf{Q}_{n \mathbf{k}}=\omega_{n \mathbf{k}} \mathbf{Q}_{n \mathbf{k}}
$$

It can be demonstrated that $\mathbf{M}_{g}^{-1} \cdot \hat{L}$ is a Hermitian operator with respect to the weighted inner product

$$
\left\langle\mathbf{Q}_{A} \mid \mathbf{Q}_{B}\right\rangle=\frac{1}{2} \int d^{3} \mathbf{r} \mathbf{Q}_{A}^{*}(\mathbf{r}) \cdot \mathbf{M}_{g}(\mathbf{r}) \cdot \mathbf{Q}_{B}(\mathbf{r})
$$

Then, according to the spectral theorem, the eigenfunctions $\mathbf{Q}_{n \mathbf{k}}$ define a complete set of basis vectors and can be normalized as

$$
\left\langle\mathbf{Q}_{m \mathbf{q}} \mid \mathbf{Q}_{n \mathbf{k}}\right\rangle=\delta_{n, m} \delta_{\mathbf{q}, \mathbf{k}}
$$

As demonstrated in Ref.[46] the above normalization condition implies that the electromagnetic field sub-component of the state vector is normalized as in Eq. (6).

Next, we introduce a Green's function $\underline{G}_{Q}$ for the generalized problem defined as the solution of:

$$
\left(\hat{L}-\omega \mathbf{M}_{g}\right) \cdot \underline{G}_{Q}\left(\mathbf{r}, \mathbf{r}_{1}\right)=i \delta\left(\mathbf{r}-\mathbf{r}_{1}\right) \mathbf{1}
$$

Evidently, the tensor $\underline{G}_{Q}$ can be expanded as $\underline{G}_{Q}\left(\mathbf{r}, \mathbf{r}_{1}\right)=\sum_{n \mathbf{k}} \mathrm{Q}_{n \mathbf{k}}(\mathbf{r}) \otimes \boldsymbol{\alpha}_{n \mathbf{k}}$ where $\boldsymbol{\alpha}_{n \mathbf{k}}$ are unknown vectorial coefficients $[4,59]$. Using the completeness of the eigenfunctions and standard ideas [59], it is readily found that:

$$
\boldsymbol{\alpha}_{n \mathbf{k}}=\frac{i}{2\left(\omega_{n \mathbf{k}}-\omega\right)} \mathbf{Q}_{n \mathbf{k}}^{*}\left(\mathbf{r}_{1}\right)
$$

Thus, it follows that the generalized Green's function has the eigenmode expansion:

$$
\underline{G}_{Q}\left(\mathbf{r}, \mathbf{r}_{1}\right)=\sum_{n \mathbf{k}} \frac{i}{2\left(\omega_{n \mathbf{k}}-\omega\right)} \mathbf{Q}_{n \mathbf{k}}(\mathbf{r}) \otimes \mathbf{Q}_{n \mathbf{k}}^{*}\left(\mathbf{r}_{1}\right)
$$

The Green's function of the original system (B1) is the restriction of $\underline{G}_{Q}$ to its first six by six components:

$$
\underline{G}\left(\mathbf{r}, \mathbf{r}_{1}, \omega\right)=\sum_{n \mathbf{k}} \frac{i}{2\left(\omega_{n \mathbf{k}}-\omega\right)} \mathbf{F}_{n \mathbf{k}}(\mathbf{r}) \otimes \mathbf{F}_{n \mathbf{k}}^{*}\left(\mathbf{r}_{1}\right)
$$


with $\mathbf{F}_{n \mathbf{k}}(\mathbf{r})$ normalized as in Eq. (6).

[1] K. H. Drexhage, "Monomolecular Layers and Light," Scientific American, vol. 222, pp. 108119, Mar. 1970.

[2] K. H. Drexhage, "IV Interaction of Light with Monomolecular Dye Layers," in Progress in Optics (E. Wolf, ed.), vol. 12, pp. 163-232, Elsevier, 1974.

[3] R. R. Chance, A. Prock, and R. Silbey, "Molecular Fluorescence and Energy Transfer Near Interfaces," in Advances in Chemical Physics (I. Prigogine and S. A. Rice, eds.), pp. 1-65, John Wiley \& Sons, Inc., 1978.

[4] L. Novotny and B. Hecht, Principles of Nano-Optics. Cambridge: Cambridge University Press, 2 edition ed., Nov. 2012.

[5] S. A. Fulling and P. C. W. Davies, "Radiation from a Moving Mirror in Two Dimensional Space-Time: Conformal Anomaly," Proceedings of the Royal Society of London A: Mathematical, Physical and Engineering Sciences, vol. 348, pp. 393-414, Mar. 1976.

[6] J. Schwinger, "Casimir energy for dielectrics," Proceedings of the National Academy of Sciences, vol. 89, pp. 4091-4093, Jan. 1992.

[7] G. Barton and C. Eberlein, "On Quantum Radiation from a Moving Body with Finite Refractive Index," Annals of Physics, vol. 227, pp. 222-274, Nov. 1993.

[8] G. Barton, "The Quantum Radiation from Mirrors Moving Sideways," Annals of Physics, vol. 245, pp. 361-388, Feb. 1996.

[9] C. Eberlein, "Sonoluminescence as Quantum Vacuum Radiation," Physical Review Letters, vol. 76, pp. 3842-3845, May 1996.

[10] V. V. Dodonov, "Current status of the dynamical Casimir effect," Physica Scripta, vol. 82, no. 3, p. 038105, 2010.

[11] S. I. Maslovski and M. G. Silveirinha, "Quantum friction on monoatomic layers and its classical analogue," Phys. Rev. B, vol. 88, p. 035427, 2013.

[12] M. F. Maghrebi, R. Golestanian, and M. Kardar, "Scattering approach to the dynamical Casimir effect," Physical Review D, vol. 87, p. 025016, Jan. 2013.

[13] M. G. Silveirinha, "Theory of quantum friction," New Journal of Physics, vol. 16, p. 063011, June 2014. 
[14] G. Pieplow and C. Henkel, "Cherenkov friction on a neutral particle moving parallel to a dielectric," J. Phys. Condens. Matter., vol. 27, p. 214001, 2015.

[15] M. G. Silveirinha, "Quantization of the electromagnetic field in nondispersive polarizable moving media above the Cherenkov threshold," Physical Review A, vol. 88, p. 043846, Oct. 2013.

[16] P. P. Meyer, "A quantum Cerenkov effect," Journal of Physics A: Mathematical and General, vol. 18, no. 12, p. 2235, 1985.

[17] E. V. Teodorovich, "On the Contribution of Macroscopic Van Der Waals Interactions to Frictional Force," Proceedings of the Royal Society of London A: Mathematical, Physical and Engineering Sciences, vol. 362, pp. 71-77, June 1978.

[18] L. S. Levitov, "Van Der Waals' Friction," EPL (Europhysics Letters), vol. 8, no. 6, p. 499, 1989.

[19] V. E. Mkrtchian, "Interaction between moving macroscopic bodies: viscosity of the electromagnetic vacuum," Physics Letters A, vol. 207, pp. 299-302, Nov. 1995.

[20] J. B. Pendry, "Shearing the vacuum - quantum friction," Journal of Physics: Condensed Matter, vol. 9, p. 10301, Nov. 1997.

[21] A. I. Volokitin and B. N. J. Persson, "Theory of friction: the contribution from a fluctuating electromagnetic field," Journal of Physics: Condensed Matter, vol. 11, no. 2, p. 345, 1999.

[22] A. I. Volokitin and B. N. J. Persson, "Near-field radiative heat transfer and noncontact friction," Reviews of Modern Physics, vol. 79, pp. 1291-1329, Oct. 2007.

[23] S. Scheel and S. Y. Buhmann, "Casimir-Polder forces on moving atoms," Physical Review A, vol. 80, p. 042902, Oct. 2009.

[24] T. G. Philbin and U. Leonhardt, "No quantum friction between uniformly moving plates," New Journal of Physics, vol. 11, p. 033035, Mar. 2009.

[25] J. B. Pendry, "Quantum friction - fact or fiction?," New Journal of Physics, vol. 12, p. 033028, Mar. 2010.

[26] U. Leonhardt, "Comment on 'Quantum Friction-Fact or Fiction?'," New Journal of Physics, vol. 12, no. 6, p. 068001, 2010.

[27] J. B. Pendry, "Reply to comment on 'Quantum friction-fact or fiction?'," New Journal of Physics, vol. 12, no. 6, p. 068002, 2010.

[28] G. Barton, "On van der Waals friction. II: Between atom and half-space," New Journal of 
Physics, vol. 12, no. 11, p. 113045, 2010.

[29] G. V. Dedkov and A. A. Kyasov, "Tangential and Quantum Frictional Forces on a Neutral Particle Moving Near a Surface," Journal of Computational and Theoretical Nanoscience, vol. 9, pp. 117-121, Jan. 2012.

[30] S. A. R. Horsley, "Canonical quantization of the electromagnetic field interacting with a moving dielectric medium," Phys. Rev. A, vol. 86, p. 023830, 2012.

[31] J. S. Høye and I. Brevik, "Casimir friction between dense polarizable media," Entropy, vol. 15, p. 3045, 2013.

[32] G. Pieplow and C. Henkel, "Fully covariant radiation force on a polarizable particle," New Journal of Physics, vol. 15, no. 2, p. 023027, 2013.

[33] A. I. Volokitin and B. N. J. Persson, "Comment on 'Fully covariant radiation force on a polarizable particle'," New Journal of Physics, vol. 16, no. 11, p. 118001, 2014.

[34] J. S. Høye and I. Brevik, "Casimir friction at zero and finite temperatures," Eur. Phys. J. D, vol. 61, p. 61, 2014.

[35] K. A. Milton, J. S. Høye, and I. Brevik, "The reality of casimir friction," Symmetry, vol. 8, p. 29, 2016.

[36] J. S. Høye and I. Brevik, "Casimir friction: Relative motion more generally," J. Phys.: Condensed Matter, vol. 27, p. 214008, 2015.

[37] J. S. Høye, I. Brevik, and K. A. Milton, "Casimir friction between polarizable particle and half-space with radiation damping at zero temperature," Journal of Physics A: Mathematical and Theoretical, vol. 48, no. 36, p. 365004, 2015.

[38] M. G. Silveirinha, "Optical Instabilities and Spontaneous Light Emission by Polarizable Moving Matter," Physical Review X, vol. 4, p. 031013, July 2014.

[39] S. A. R. Horsley and S. S. Bugler-Lamb, "Negative frequencies in wave propagation: a microscopic model," Phys. Rev. A, vol. 93, p. 063828, 2016.

[40] Y. Guo and Z. Jacob, "Singular evanescent wave resonances in moving media," Optics express, vol. 22, pp. 26193-26202, 2014.

[41] S. Lannebère and M. G. Silveirinha, "Wave instabilities and unidirectional light flow in a cavity with rotating walls," Physical Review A, vol. 94, p. 033810, Sept. 2016.

[42] L. Knoll, W. Vogel, and D. G. Welsch, "Action of passive, lossless optical systems in quantum optics," Phys. Rev. A, vol. 36, p. 3803, 1987. 
[43] R. J. Glauber and M. Lewenstein, "Quantum optics of dielectric media," Phys. Rev. A, vol. 43, p. 467, 1991.

[44] N. A. R. Bhat and J. E. Sipe, "Hamiltonian treatment of the electromagnetic field in dispersive and absorptive structured media," Phys. Rev. A, vol. 73, p. 063808, 2006.

[45] M. G. Silveirinha and S. I. Maslovski, "Exchange of momentum between moving matter induced by the zero-point fluctuations of the electromagnetic field," Physical Review A, vol. 86, p. 042118 , Oct. 2012 .

[46] M. G. Silveirinha, "Chern invariants for continuous media," Physical Review B, vol. 92, p. 125153 , Sept. 2015.

[47] A. Raman and S. Fan, "Photonic Band Structure of Dispersive Metamaterials Formulated as a Hermitian Eigenvalue Problem," Physical Review Letters, vol. 104, p. 087401, Feb. 2010.

[48] T. A. Morgado, D. E. Fernandes, and M. G. Silveirinha, "Analytical Solution for the Stopping Power of the Cherenkov Radiation in a Uniaxial Nanowire Material," Photonics, vol. 2, pp. 702-718, June 2015.

[49] S. Haroche and J.-M. Raimond, Exploring the Quantum: Atoms, Cavities, and Photons. Oxford ; New York: Oxford University Press, Oct. 2006.

[50] J. D. Jackson, Classical electrodynamics. New York, NY: Wiley, 3rd ed. ed., 1999.

[51] R. Shankar, Principles of Quantum Mechanics. Boston, MA: Springer US, 1994.

[52] M. V. Nezlin, "Negative-energy waves and the anomalous Doppler effect," Soviet Physics Uspekhi, vol. 19, pp. 946-954, Nov. 1976.

[53] V. P. Frolov and V. L. Ginzburg, "Excitation and radiation of an accelerated detector and anomalous doppler effect," Physics Letters A, vol. 116, pp. 423-426, July 1986.

[54] R. Loudon and S. M. Barnett, "Theory of the linear polarizability of a two-level atom," Journal of Physics B: Atomic, Molecular and Optical Physics, vol. 39, p. S555, Aug. 2006.

[55] L. Allen and J. H. Eberly, Optical Resonance and Two-level Atoms. Courier Corporation, 1975 .

[56] F. Intravaia, R. O. Behunin, and D. A. R. Dalvit, "Quantum friction and fluctuation theorems," Physical Review A, vol. 89, p. 050101, May 2014.

[57] S. A. Maier, Plasmonics: Fundamentals and Applications. New York, NY: Springer, softcover reprint of hardcover 1st ed. 2007 edition ed., Dec. 2010.

[58] P. B. Johnson and R. W. Christy, "Optical Constants of the Noble Metals," Physical Review 
$B$, vol. 6, no. 12 , pp. 4370-4379, 1972.

[59] K. Sakoda, Optical Properties of Photonic Crystals. Berlin ; New York: Springer, 2nd edition ed., Nov. 2004. 